\title{
Nuevas consideraciones sobre la morfología verbal del cabécar ${ }^{1}$
}

\section{Guillermo González Campos ${ }^{2}$}

\author{
Universidad de Costa Rica
}

\begin{abstract}
resumen
En cabécar, el verbo es la categoría léxica que, desde la morfología flexiva, presenta más complejidad. El artículo hace resumen histórico de las propuestas de análisis hechas al respecto. Propone una forma de entender la estructura morfológica del verbo cabécar, con base en tres categorías fundamentales: la raíz verbal, los sufijos desinenciales y los clíticos verbales. A partir de ello, se hace una propuesta de paradigma verbal para esta lengua.
\end{abstract}

\section{abstract}

From the point of view of inflectional morphology, the verb is the most complex lexical category in Cabécar. This article reviews the history of research on this topic. It proposes a way to understand the morphological structure of the Cabécar verb, based on three essential categories: the verbal root, the inflectional suffixes and the verbal clitics. Then, using these elements, a proposal of verbal paradigm is developed for this language.

Palabras claves: idioma cabécar, morfología verbal, análisis lingüístico Keywords: Cabécar language, verbal morphology, linguistic analysis

1 Versión revisada de la ponencia leída en el I Simposio Internacional del Programa de Lingüística Centroamericana (PROLINCA) «Centroamérica: un microcosmo lingüístico», llevado a cabo los 7 y 8 de mayo 2012, en la ciudad de Heredia, en el campus Omar Dengo de la Universidad Nacional de Costa Rica. Recibido: 6 de noviembre de 2011; aceptado: 6 de enero de 2012.

2 Sede del Atlántico. Correo electrónico: gonzalezcampos@gmail.com 


\section{Introducción}

Este artículo desarrolla una nueva propuesta de descripción de la morfología verbal del cabécar, lengua indígena costarricense perteneciente a la familia chibcha. Tal categoría léxica es la que más cambios presenta, por lo que el análisis y sistematización de las diferentes formas verbales es un paso para la comprensión de los principales fenómenos gramaticales de cabécar. Se hace un recuento de las anteriores investigaciones que han tratado este tema y se analizan los paradigmas verbales planteados por ellas. Esto se lleva a cabo con dos propósitos: señalar los problemas que tienen estos modelos y explicitar las razones por las cuales es necesario un nuevo análisis de la estructura morfológica del verbo cabécar. Se texto incluye una descripción pormenorizada de los elementos que se encuentran involucrados en la formación de dichos verbos. Además, se presentan diferentes paradigmas que incluyen tanto la sistematización de los sufijos flexivos involucrados en este proceso como ejemplos de formas conjugadas.

\section{Estudios previos}

Hasta ahora existen cuatro propuestas de descripción de la morfología verbal del cabécar. El primer intento de dar una visión global de la conjugación de esta lengua es de Víctor Manuel Arroyo Soto (1921-1975), en su tesis de licenciatura de 1950, posteriormente editada como libro ${ }^{3}$. Esta obra, pionera en el estudio de las lenguas indígenas del país, ofrece una descripción básica de cuatro idiomas: el boruca, el térraba, el cabécar y el bribri ${ }^{4}$. En todos los casos, esta sinopsis gramatical incluye la exposición de la morfología verbal. Desdichadamente, esta se hizo tomando como referencia las formas verbales del español, las

3 Víctor Manuel Arroyo Soto, Lengua indígenas costarricenses (San José: Editorial Costa Rica, 1966) 136-147.

4 Dado su estrecho parentesco, estas dos últimas lenguas fueron estudiadas de forma conjunta por el autor. 
cuales, claro está, no constituyen un buen referente para la comprensión del sistema verbal de las lenguas indígenas de Costa Rica.

Las ocho categorías verbales propuestas por Arroyo se incluyen en el cuadro 1, el cual incluye además un ejemplo que las ilustra. En la trascripción, se respeta la ortografía utilizada por el autor.

\section{Cuadro 1. Formas verbales propuestas para el cabécar por Arroyo}

\begin{tabular}{|c|c|c|}
\hline \multicolumn{2}{|c|}{ Formas Verbales } & $\begin{array}{l}\text { Ejemplo } \\
\text { 'caminar' }\end{array}$ \\
\hline \multirow{2}{*}{\multicolumn{2}{|c|}{$\begin{array}{c}\text { infinitivo } \\
\text { preSente }\end{array}$}} & ske \\
\hline & & skegé \\
\hline \multirow{2}{*}{\multicolumn{2}{|c|}{$\begin{array}{r}\text { pretérito } \\
\text { futuro }\end{array}$}} & ská \\
\hline & & ma ske \\
\hline \multicolumn{2}{|r|}{ imperativo } & $\begin{array}{l}\text { ba ske } \\
\text { bas ske }\end{array}$ \\
\hline \multirow{3}{*}{ participio } & paSivo & skelejka \\
\hline & activo & skabí \\
\hline & Gerundio & skagé \\
\hline
\end{tabular}

Uno de los principales problemas de ese trabajo es la trascripción incorrecta de los sonidos del cabécar. El primer fonema de la raíz verbal de 'caminar' no es /s/, sino / $/$ /. Por otro lado, es inadecuado categorizar en términos de «tiempos» las formas verbales del cabécar, pues estas representan más bien nociones de tipo aspectual y modal. Así, lo que este autor llama «presente» es, en realidad, la forma de aspecto imperfectivo y lo que denomina «pasado» constituye el aspecto perfectivo. Además, la equivocada tendencia de tomar el español como referente para juzgar los hechos del cabécar hace que se «identifiquen» formas verbales que no existen en esta lengua. Tal es el caso de las categorías de «participio»y «gerundio», inexistentes en el sistema verbal de 
dicho idioma ${ }^{5}$. Por ejemplo, la palabra shkë lë jka, que erróneamente se da como un «participio pasivo» es una forma de aspecto retrospectivo (que, como se verá, se marca con el sufijo -l -lé ) a la cual se le ha agregado el morfema $j k a$. La confusión se debe a que los cabécares suelen traducir esta categoría verbal al español mediante el pretérito perfecto compuesto y el pretérito pluscuamperfecto, perífrasis verbales construidas precisamente a través de un participio pasivo. Una confusión similar ocurre con el «participio activo». La palabra shké bil es un sustantivo que significa 'andariego, persona aficionada a caminar' y el sufijo -bíl -lbí no es de tipo flexivo, sino derivativo.

La segunda propuesta de descripción global del verbo en cabécar se debe a David Bourland (1928-2000) ${ }^{6}$, lingüista estadounidense que en la década de 1970 trabajó en la Universidad de Costa Rica e hizo investigaciones sobre el cabécar de Ujarrás. Publicó en 1974 el único estudio global de la sintaxis del cabécar hecho hasta el momento, empleado como base teórica la primera versión del modelo generativo transformacional propuesto por Noam Chomsky?. Este primer intento de descripción del cabécar basado en una teoría lingüística moderna fue casi por completo fallido. Al igual que en el caso de Arroyo, la obra presenta deficiencias en lo que respecta a la transcripción de los sonidos del cabécar, especialmente en lo atinente al tono, que Bour-

5 Esta tendencia de «meter» al cabécar dentro de la gramática española se ha mantenido hasta la actualidad. Durante el trabajo de campo con maestros cabécares, hemos constatado que estos desconocen la realidad lingüística de su propio idioma y, en las clases, transmiten interpretaciones erradas sobre este. Muchas de estas malas interpretaciones se han solapado en obras didácticas. Este el caso de la serie Sa ña yuwá sa siwa wa. (Según los cánones ortográficos actuales, este título está escrito de forma incorrecta. La forma correcta es Sá ña yuwá sá sị wá wa, que significa «Nos formamos con nuestro conocimiento tradicional».)

6 Bourland es más conocido por su trabajo como semanticista. Tras su regreso a los Estados Unidos en 1984, se dedicó al desarrollo del E-Prime (English-Prime), modelo de escritura del inglés en el que se prescinde de las formas del verbo to be. Él había hecho esta propuesta en la década de 1970, con base en la semántica general de Alfred Korzybski, de quien había sido alumno.

7 David Bourland Hawley, A Generative-Transformational Grammar of an Idiolect of Cabécar (San José, C.R.: Imprenta Semántica, 1974). Existe una traducción al español de esta obra: «Una gramática generativo-transformacional del cabécar», Revista de Filología y Lingüística de la Universidad de Costa Rica 2(3) (1976): 49-112. 
land nunca reconoció. En relación con el verbo, la obra hace un intento de descripción del sistema TAM del cabécar, pero con pocos aciertos. Según este autor, hay en cabécar tres modos (indicativo, subjuntivo y habitual), dos voces (activa y pasiva), dos tiempos (presente y pasado) y dos formas aspectuales ([ + imperf y [ - imperf $]$ ). Como se verá luego, ninguna de estas apreciaciones es correcta.

En el cuadro 2, se presenta el paradigma de conjugación verbal elaborado por este autor. Se presentan dos ejemplos, con el fin de ilustrar las dieciséis formas verbales propuestas por él. Al igual que en el caso anterior, se respeta la ortografía original.

\section{Cuadro 2. Formas verbales propuestas para el cabécar por Bourland}

\begin{tabular}{|c|c|c|}
\hline \multirow{2}{*}{ Formas verbales } & \multicolumn{2}{|c|}{ Ejemplos } \\
\hline & 'dar' & 'hacer' \\
\hline infinitivo & memí & zuwó \\
\hline $\begin{array}{r}\text { Presente } \\
\text { afirmativo } \\
\text { neGativo } \\
\text { interroGativo } \\
\text { habitual } \\
\text { paSivo }\end{array}$ & $\begin{array}{l}\text { męgę } \\
\text { menitt } \\
\text { Imegé ItI } \\
\text { megęé-LU }\end{array}$ & $\begin{array}{l}\text { izuwegę } \\
\text { kạ wa ... izúna }\end{array}$ \\
\hline $\begin{array}{r}\text { afirmativo } \\
\text { neGativo } \\
\text { habitual } \\
\text { paSivo } \\
\end{array}$ & $\begin{array}{l}\text { imá } \\
\text { Imegé } \\
\text { imená-LU }\end{array}$ & $\begin{array}{l}\text { te... izuwä } \\
\text { ká... suwá ... izúne }\end{array}$ \\
\hline $\begin{array}{r}\text { afirmativo } \\
\text { interroGativo }\end{array}$ & $\begin{array}{l}\text { męgę́ LonI } \\
\text { Imemit }\end{array}$ & \\
\hline $\begin{array}{r}\text { afirmativo } \\
\text { neGativo }\end{array}$ & 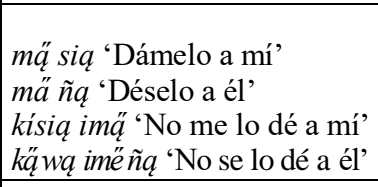 & $\begin{array}{l}\text { izuwúbugę } \\
\text { kéi zųwá }\end{array}$ \\
\hline $\begin{array}{lr}\text { Progresivo } & \\
& \begin{array}{r}\text { imperfectivo } \\
\text { perfectivo }\end{array} \\
\end{array}$ & $\begin{array}{l}\text { káLひ imä } \\
\text { megę na }\end{array}$ & \\
\hline
\end{tabular}


Además de los problemas de transcripción señalados, Bourland tuvo dificultades para establecer la correcta segmentación de los morfemas verbales. Por ejemplo, la $i$ que en el cuadro se coloca antes de muchas formas verbales no forma parte de la raíz verbal ni constituye un elemento propio de la conjugación. Se trata de una forma proclítica cuya función es «ocupar» el espacio de la función sintáctica denominada absolutivo. En cabécar, muchas formas verbales requieren obligatoriamente la presencia de esta categoría; por eso, cuando no se desea explicitarla mediante una frase nominal, debe colocarse el pronombre $i$ en su lugar. Otro caso es el de $s$ ' $\underline{i} a$ 'a mí', frase posposicional formada por la forma reducida del pronombre yís 'yo' seguida de la posición $\underline{i} a$, que marca el dativo. Parece claro que este elemento no es un constituyente morfológico del verbo; sin embargo, por la colocación que Bourland hace lo interpreta como una desinencia. Situación similar se presenta en la categoría denominada por él «presente habitual». Coloca en ese espacio una oración completa que no supo segmentar correctamente:

$i \quad i$ të. Finalmente, habría que señalar la tendencia de este autor mége

de categorizar las formas verbales mediante nociones temporales como «presente» o «pasado», lo cual, como se indicó antes, no es adecuado para una lengua como el cabécar.

El tercer intento de ofrecer una explicación general de la morfología verbal del cabécar se encuentra en el trabajo de Aziel W. Jones, misionero estadounidense que visitó la región cabécar de Chirripó en 1952. Aunque no lo indica, es claro que su propuesta parte del modelo morfológico conocido con el nombre de ítem y diSpoSición (en inglés, Item and Arrangement), el cual, como se sabe, supone que las palabras se forman por medio de una combinatoria (arrangement) de diversos morfemas (items). Basado en estos supuestos, Jones propuso, en un primer trabajo, que todo verbo cabécar se compone de una raíz seguido de un «sufijo obligatorio de tiempo». Además, estableció que todo verbo posee dos raíces, que denominó «A»y «B». La primera, según él, se utiliza para la construcción de tiempos 
o aspectos más «sencillos», mientras que la segunda se utiliza para 
aquellos que son «complejos» ${ }^{8}$. Posteriormente, en otro artículo, Jones estableció que la raíz verbal constituía un «núcleo» que puede ser expandido por medio de «satélites» (sufijos y prefijos $\left.{ }^{9}\right)$. Los sufijos pueden ser de tres tipos: de primer orden, obligatorios y transportan el significado aspectual temporal; de segundo orden, potestativos y señalan el «tipo de acción del verbo», como -qué (actualmente transcrito -ké ), y de tercer orden, clasificación que, según este autor, comprende cinco sufijos direccionales opcionales: -te, -mí, wá, -cá

$\mathrm{y}-\operatorname{cs} a^{10}$. Este autor reconoce como fundamentales solo los primeros y propone el modelo de conjugación verbal expuesto en el cuadro 3.

\section{Cuadro 3. Formas verbales propuestas para el cabécar, según Jones}

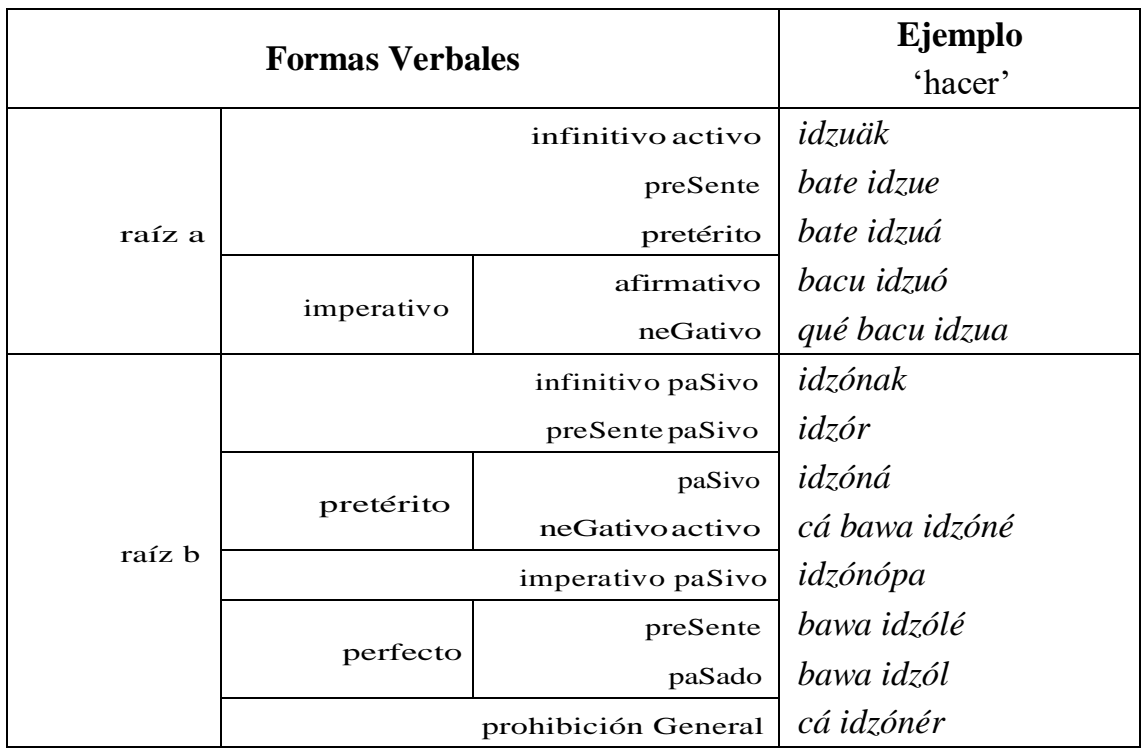

8 AzielW.Jones, «La clave del verbo en la lengua cabécar», América Indígena 34(2) (1974): 333-339.

9 El cabécar, en realidad, no posee prefijos. De forma errónea, Jones considera prefijos a pronombres y sustantivos que, por ocupar la posición de absolutivo, se colocan antes del verbo.

10 Aziel W. Jones, «El verbo cabécar: núcleo con satélites», América Indígena 43(1) (1983): 149154. Tal como se ha hecho, se respeta la ortografía utilizada por el autor. 
$\mathrm{Al}$ igual que en los casos anteriores, Jones incurre en diversos errores de transcripción, aunque no tan pronunciados como los de los autores antes vistos. Tuvo dificultades con el fonema $/ \mathrm{I} /$, que confundió con /e/, y con / $/$, el cual en algunas ocasiones lo representó con $u$ y en otras, con $o$. También presenta errores tanto en la colocación de la marca de nasalidad (que en su artículos es representada mediante el formato tipográfico de cursiva) y de la tilde que señala el tono alto. También es notable su tendencia a escribir las posposiciones pegadas a las palabras que rigen cual si fueran sufijos. Nótese que escribe bate, bacu y bawa en lugar de bá te, bá $\underline{k} u$ y bá wa $a$. Su propuesta, además, es hereda de la tendencia anterior de utilizar una nomenclatura de tiempos verbales, lo cual, como ya se mencionó, no es conveniente para el cabécar. Asimismo, al igual que Bourland, se equivoca al suponer que el proclítico $i$ constituye parte del paradigma verbal. Finalmente, Jones se equivoca al considerar como diferentes las formas verbales yöl y yölë. Se trata simplemente de variantes dialectales de la misma palabra.

Debe reconocerse, no obstante, que la segmentación de los morfemas hecha por él es, en términos generales, bastante acertada. $\mathrm{Su}$ idea de que todo verbo posee dos raíces es correcta en el modelo teórico dentro del cual trabaja y, aunque su caracterización nocional de las formas verbales del cabécar no es del todo acertada, la identificación de los sufijos flexivos es, en cierta medida, aceptable. Importante resulta sobre todo su consideración de que los elementos que él caracteriza como de segundo y tercer orden no forman parte del paradigma de conjugación. Puede decirse, por lo tanto, que el trabajo de este autor constituyó un primer paso en el entendimiento del sistema verbal del cabécar.

La última descripción de la estructura morfológica del verbo cabécar es obra de Enrique Margery y fue publicada como artículo en $1985^{11}$ y luego incluida como parte del «Esbozo de la gramática

T1 Enrique Margery Peña, «Morfología flexiva del verbo cabécar», Estudios de Lingüística Chibcha 4 (1985): 101-154. 
cabécar» que encabeza el diccionario de este idioma que el autor elaboró ${ }^{12}$. Aunque él no lo indica, la teoría morfológica que inspira su trabajo es la conocida por el nombre de ítem y proceso (en inglés, Item and Process $)^{13}$. Esta, como su nombre lo indica, sugiere que las palabras y sus variantes se forman mediante diversas reglas (processes) que modifican una forma base (item). Para el caso particular del cabécar, Margery consideró que la forma base a partir de la cual se derivarían las demás es el infinitivo (como se verá luego, esta no fue una decisión acertada). De acuerdo con su descripción, el infinitivo se forma a partir de una o varias raíces y una vocal temática, que puede ser oral o nasal. Existen, además, una serie de sufijos que es factible agregarle al infinitivo según su significación y se mantienen al flexionar el verbo. De ellos, citó seis: $-\underline{j u} \sim \underline{u},-k a,-g \ddot{o},-t \ddot{e},-m \underline{i} \mathrm{y}$ $-s \underline{a} \sim-k s \underline{a}$. Un infinitivo verbal como bikö 'cortar', se compone de una raíz bik- y una vocal temática -ö. A partir de esta forma infinitiva, se van derivando mediante distintos procesos todas las restantes formas verbales. Lo que él denomina el «imperfecto indefinido» se deriva del infinitivo cambiando la vocal temática por -é o -é, según sea esta oral o nasal. De forma semejante, el «perfecto irremotospectivo» se forma sustituyendo la vocal temática por $-a ́ a$ o $-\underline{a}$. Otras formas se derivan a su vez de éstas. El aspecto habitual, por ejemplo, se forma agregando -ké $\sim-g e ́$ al «imperfecto indefinido»y el «antepresente» se construye al adicionar $-j k a$ al «perfecto irremotospectivo». Proce- diendo de esta forma, Margery describe un sistema que, entre formas simples y compuestas, comprende veintisiete tipos diferentes de fle- xión, las cuales han sido sistematizadas en el cuadro 4 .

12 Enrique Margery Peña, Diccionario cabécar-español español-cabécar (San José: Editorial de la Universidad de Costa Rica, 1989) lxx-cii.

13 Sobre las nociones de ítem y diSpoSición e ítem y proceso, véase la revisión que se hace en Mark Aronoff y Kirsten Fudeman, What is Morphology? (Chichester, West Sussex: Blackwell, 2011) 49-54. 


\section{Cuadro 4. Formas verbales propuestas para el cabécar por Margery}

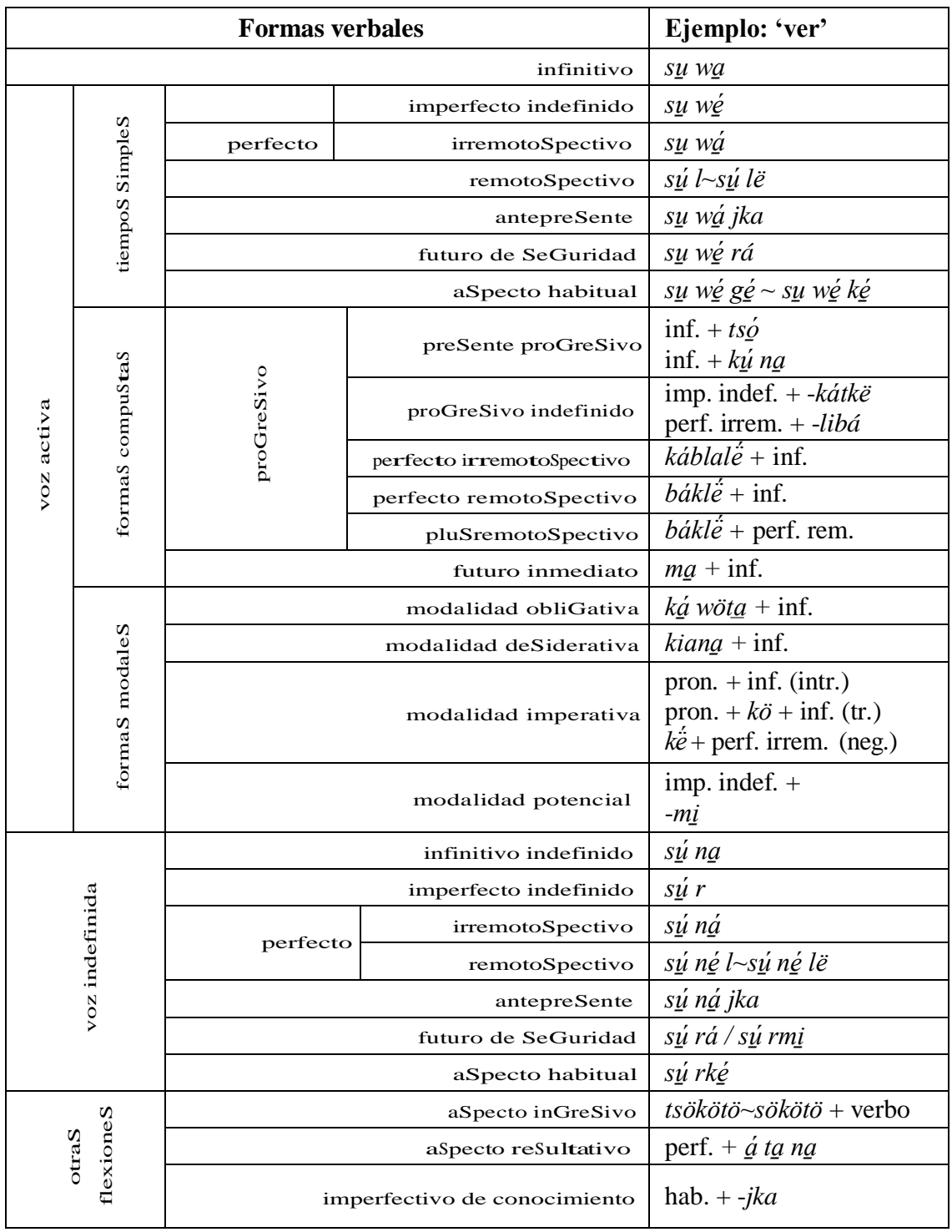


La propuesta de Margery es la mejor fundamentada desde el punto de vista lingüístico. Basada en un análisis riguroso y una cuidadosa recopilación de datos, su sistematización constituye la principal fuente de información de que se dispone actualmente para conocer la morfología verbal, pero no está exenta de problemas. En cuanto a las formas simples, dado que el tratamiento de las perífrasis verbales desborda el cometido de este trabajo, puede afirmarse que la principal desventaja del modelo propuesto por Margery es la multiplicación innecesaria de formas verbales. No hay necesidad de crear categorías como «futuro de seguridad», «aspecto habitual» o «progresivo indefinido», por citar tres casos, cuando es claro que se trata de derivaciones de lo que él denomina «imperfecto indefinido». En tales casos, la adición del elemento morfológico agrega solo un matiz de sentido sin modificar el sentido sustancial de la forma verbal (en este caso, el aspecto imperfectivo); no corresponde darles un tratamiento diferenciado. En dichos casos, más acertada es la propuesta de Jones al considerar tales elementos como formas de segundo y tercer orden, que, por lo tanto, no deben considerarse parte del paradigma verbal fundamental.

De forma general, puede decirse que el estudio de la morfología verbal del cabécar tuvo en sus inicios diversos problemas que impidieron una cabal comprensión. Principalmente, se incurrió en errores de transcripción, equivocaciones en la segmentación de los morfemas y malas interpretaciones causadas por la tendencia a analizar los hechos de este idioma a la luz de las categorías gramaticales del español. Con el tiempo, estos yerros fueron subsanándose en gran medida; actualmente las investigaciones lingüísticas del cabécar deben centrarse en una revisión y reinterpretación de hechos gramaticales ya estudiados que permitan generar una visión más sistemática de la flexión verbal de esta lengua. 


\section{Estructura del verbo en cabécar}

Teniendo en cuenta lo expuesto y considerando algunos datos nuevos recopilados ${ }^{14}$, puede defenderse la idea de que el verbo en cabécar tiene una estructura compuesta por tres tipos de elementos morfológicos: 1. la raíz verbal, que contiene el sentido léxico primario del verbo, 2. las desinencias o sufijos flexivos marcadores de las formas verbales primarias y 3 . los clíticos verbales. Considerando lo explicado antes, la forma verbal $i$ ksámi 'empezó a cantar', desde el punto de vista morfológico, se analiza de la siguiente manera: $i=k s$ $\dot{a}=m \underline{i}$ (3-cantar-pfv-inc). La raíz verbal y las desinencias conforman la estructura morfológica primaria del verbo, a la cual se agregan, de forma opcional, los clíticos verbales. Gráficamente, se representar esta situación en la figura 1.

Figura 1. Estructura de las formas verbales del cabécar



Acontinuación, se abordará en detalle cada uno de estos elementos.

\section{La raíz verbal}

Es la parte fundamental del verbo, el núcleo que transporta su significado léxico primario. Tal como planteó Jones, todo verbo en

14 Los datos aquí ofrecidos corresponden a la variedad de cabécar hablada en Chirripó, región en la que el autor de este trabajo ha investigado desde hace cuatro años. La propuesta de paradigma verbal aquí ofrecida fue revisada con informantes de cada una de las restantes regiones cabécares. 
cabécar tiene dos raíces, a las cuales es mucho más conveniente dar el apelativo de raíz débil y raíz fuerte, en lugar de la designación por letras que sugiere. La raíz débil permite construir las formas verbales más comunes (el infinitivo, el imperfectivo, el perfectivo y el imperativo), mientras que la raíz fuerte se utiliza para establecer formas verbales semánticamente más complejas (el aspecto retrospectivo y las flexiones de voz media). Jones señaló que no era factible predecir una raíz a partir de otra, pues no había un procedimiento regular de derivación, sino varios: cambio de tono, cambio de tono más cambio de vocal, «vocal agregada», etc. Igual opinión es la de Margery al explicar la formación de lo que él denominó el «perfecto remotospectivo»; según él, la cantidad y variedad de procesos morfológicos involucrados en la creación de dicho tema, hacen inútil cualquier intento de sistematizar dicha flexión.

No obstante, el proceso se torna mucho más simple y regular si, siguiendo una propuesta hecha por Adolfo Constenla para el bribri, se asume que la forma primaria del verbo es la raíz fuerte, en lugar de la raíz débil. Propone que la forma verbal básica del bribri se obtiene eliminando el saltillo de lo que él denomina «perfecto improspectivo afirmativo activo». Así, a partir de una forma bribri como $y \ddot{o}$ ' es factible obtener el tema $y \ddot{o}^{-15}$. En cabécar no existe esta forma aspectual, pero el tema puede obtenerse suprimiendo el sufijo -l -lé de la forma verbal de aspecto retrospectivo (la cual, como se dijo antes, Margery denomina «perfecto remotospectivo») ${ }^{16}$. Así, de forma similar al bribri, a partir de yölé, puede obtenerse yö-, el tema verbal denominado aquí raíz fuerte.

Una vez procedido de esta forma, puede derivarse la raíz débil a partir de la raíz fuerte mediante dos procesos fundamentales:

15 Adolfo Constenla, Feliciano Elizondo y Francisco Pereira, Curso básico de bribri (San José: Editorial de la Universidad de Costa Rica, 1998) 35-37.

16 También puede obtenerse la raíz eliminando las desinencias de cualquiera de las formas verbales de voz media. 
a) Cuando la raíz fuerte termina en á, é y é , la raíz débil se forma suprimiendo dicha $\operatorname{vocal}^{17}$, como se muestra en los ejemplos incluidos en el cuadro 5 .

\section{Cuadro 5. Ejemplos de formación de la raíz débil} a partir de la sustracción de la vocal de la raíz fuerte

\begin{tabular}{|l|c|l|}
\hline Raíz fuerte & Raíz fébil \\
\hline$\tilde{n} \underline{a}-$ & $\tilde{n}-$ & 'comer' \\
$y a ́-$ & $y-$ & 'beber' \\
$y a k a ́-$ & $y a k-$ & 'comer' \\
$k a p \ddot{e}-$ & $k a p-$ & 'dormir' \\
$k s \tilde{e}-$ & $k s-$ & 'cantar' \\
$t k \ddot{e}-$ & $t k-$ & 'punzar' \\
$m \underline{e}-$ & $m-$ & 'dar' \\
\hline
\end{tabular}

b) Cuando la raíz fuerte termina ó,ô, í, ú y ú, dicha vocal pierde el tono alto y, dado que los sufijos desinenciales que se le agregan son vocálicos, esta se vuelve una semiconsonante ([w] o [j], según corresponda) para formar la raíz débil. En el cabécar sureño, además, suele darse la anaptixis de la vocal $u$ en las secuencias [Cw-], lo cual da como resultado [Cuw-]. Ambos fenómenos se puede apreciar en los casos presentados en el cuadro 6.

17 Según esto, la raíz yá- no se forma a partir de $y$ - por medio de la inserción de á. Al contrario, $y$ - se deriva de yá- suprimiendo dicha vocal. Nótese que esta propuesta es mucho más económica y simple que las hechas por Jones y Margery. Jones, al tomar la raíz débil como primaria, debía suponer en estos casos la existencia una vocal agregada que era imposible de predecir. Margery, al proceder de forma semejante (pues el infinitivo se forma con la raíz débil), debía suponer varios cambios en la vocal temática, los cuales tampoco son factibles de sistematizarse. 


\section{Cuadro 6. Ejemplos de formación de la raíz débil}

a partir de la pérdida del tono alto de la vocal de la raíz fuerte y la consecuente conversión a semiconsonante

\begin{tabular}{|c|c|c|}
\hline Raíz Fuerte & Raíz Débil & \\
\hline$k o^{\prime}-$ & $k \underline{u}-[\mathrm{k} \tilde{\mathrm{w}}-]$ & 'encontrar' \\
\hline$o^{\prime}-$ & $w-[\tilde{\mathrm{W}}-]$ & 'hacer' \\
\hline$\underline{t o}-$ & $t \underline{u} u-\left[\mathrm{t}^{\mathrm{h}} \mathrm{w}-\right] / \underline{t a} w-$ & 'comprar' \\
\hline$k \dot{o}-$ & $k u-[\mathrm{kw}-]$ & 'morder' \\
\hline$y \ddot{o}-$ & $y u-[\mathrm{d} z \mathrm{w}-] / y u w-$ & 'hacer' \\
\hline$b i^{\prime}$ & $b i-[\mathrm{bj}-]$ & 'escarbar' \\
\hline$k i-$ & $k i-[\mathrm{kj}-]$ & 'llamar' \\
\hline$b u^{-}$ & $b u-[\mathrm{bw}-]$ & 'doblar' \\
\hline$\dot{u}-$ & $w-$ & 'moler' \\
\hline$s \underline{u}-$ & $s \underline{u}-[\mathrm{s} \tilde{\mathrm{W}}-] / s \underline{u} w-$ & 'ver' \\
\hline
\end{tabular}

Conviene indicar que el proceso descrito tiene algunas excepciones explicables en la diacronía de la lengua. La primera regla no se cumple con la raíz të- 'cortar', la cual, a pesar de terminar en vocal ë, forma la raíz débil por medio de la conversión de la vocal a la semiconsonante [j]. Además, existe una serie de verbos que presentan supletivismo. Este es el caso de utë- 'hablar' y buká- 'golpear', cuyas raíces débiles son $k t$-/tt- y $k p-/ p p$-, respectivamente. Existen, finalmente, una serie de verbos que, en apariencia, no cambian la raíz. Tal es el caso de já - 'botar' o pí- 'freír'. Estas raíces, en realidad, se conjugan mediante la formación de un verbo compuesto. Los infinitivos já wa y píwa están constituidos por las raíces anteriores

más el verbo $w \underline{a}$ 'hacer', cuya raíz primaria es $\underline{o}-$. En las variedades sureñas de la lengua, dicha raíz tiende a conservarse en el retrospectivo. De esta forma, se presentan las formas já o 'lée y púo 'lée, respec-

tivamente. No obstante, en Chirripó, la vocal $o$ cae en estos casos, originando jó lë y pílë. 


\section{Los sufijos desinenciales}

Su principal función en el cabécar es especificar la forma verbal en que se encuentra conjugado el verbo. En cabécar existen dos conjugaciones: la oral y la naSal. La conjugación oral, obviamente, utiliza vocales temáticas orales, mientras que la nasal utiliza vocales nasales. Cada forma verbal del cabécar se caracteriza por tener una desinencia específica. El infinitivo verbal, por ejemplo, utiliza la vocal temática $-\ddot{a} /-\ddot{o}$ en la conjugación oral y $-a$ en la conjugación nasal ${ }^{18}$. De forma semejante, el aspecto perfectivo se caracteriza por presentar la vocal $-\dot{a} \sim \underline{a}$, mientras que el imperativo se caracteriza

más bien por utilizar el sufijo $-\ddot{O} /-\underline{o}$.

Las categorías fundamentales para comprender la conjugación del verbo son, en primer lugar, la voz, que expresa la forma como se establecen las relaciones entre el verbo y las entidades involucrados en la acción; en segundo lugar, el aspecto, que categoriza la forma verbal según diferentes perspectivas (inicio, continuidad, reiteración, conclusión, etc.) asociadas a la percepción que tiene el hablante del desarrollo del evento denotado por el verbo. En este sentido, el cabécar se diferencia notoriamente del español, que flexiona el verbo sobre la base de la categoría denominada «tiempo verbal». En cabécar existen estrategias gramaticales para señalar cuándo tuvo o tendrá lugar una acción, pero no constituyen la base a partir del cual se estructura la conjugación del verbo. Dada esta situación, para efectos de este trabajo se considerarán como fundamentales las siguientes cinco categorías flexivas: 1 . infinitivo, 2 . imperfectivo, 3 . perfectivo, 4. imperativo y 5. retroSpectivo.

18 Los infinitivos verbales del cabécar se ven afectados por una variación dialectal. A diferencia de lo que ocurre en el sur, el cabécar norteño posee un fonema vocálico más $(/ / /)$, el cual suele transcribirse por medio del grafema $<\ddot{a}>$. En los dialectos sureños, esta vocal se fundió con $/ \mho /$. Por eso, las palabras que en el norte se pronuncian con $/ \gamma /$, en el sur se pronuncian con $/ \mho /$. Consecuentemente, los infinitivos de la conjugación oral en cabécar norteño se marca con - $\ddot{a}$ y en el sureño, con $-\ddot{o}$. En este trabajo, las variantes dialectales se separan mediante una barra inclinada $(/)$. 
Hay en cabécardos voces: la activa y la media. La activa se utiliza para señalar explícitamente que existe una entidad que realiza, ejecuta o controla la acción del verbo. Con excepción del aspecto retrospectivo en cabécar, la flexión de voz activa se construye tomando como base la raíz débil del verbo, a la cual se le agregan diferentes sufijosflexionales según la forma verbal que se quiera construir (ver cuadro 7).

\section{Cuadro 7. Desinencias verbales de la voz activa en cabécar que se agregan a la raíz débil}

\begin{tabular}{|c|c|c|c|c|c|}
\hline & \multicolumn{2}{|c|}{ Transitivo } & \multicolumn{2}{|c|}{ Intransitivo } \\
\hline & & Oral & Nasal & Oral & Nasal \\
\hline \multirow{4}{*}{ 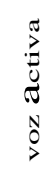 } & infinitivo & $-\ddot{a} /-\ddot{o}$ & $\underline{-a}$ & $-\ddot{a} /-\ddot{o}$ & $-a$ \\
\hline & imperfectivo & - & $\underline{-e}$ & $-\ddot{a} /-\ddot{o}$ & $-\underline{a}$ \\
\hline & perfectivo & $-\hat{a}$ & $-\underline{a}$ & $-a ́$ & $-\underline{a}$ \\
\hline & imperativo & $-\ddot{O}$ & $-\underline{o}$ & $-\ddot{o}$ & $-\underline{o}$ \\
\hline
\end{tabular}

Conforme al cuadro 7, existe una diferencia entre los verbos transitivos y los intransitivos en la forma verbal del imperfectivo. De esta forma, en cabécar, la terminación de dicha forma aspectual es - $-\ddot{e} \sim-\underline{e}$ para los verbos transitivos y $-\ddot{a} / \ddot{o}_{-}-\dot{a}$, para los intransitivos. En el cuadro 8 , se ejemplifica la conjugación en voz activa del verbo cabécar.

\section{Cuadro 8. Ejemplo de conjugación verbal de la voz activa en cabécar construidas a partir de la raíz débil}

\begin{tabular}{|c|c|c|c|c|c|}
\hline & \multicolumn{2}{|c|}{ Transitivo } & \multicolumn{2}{|c|}{ Intransitivo } \\
\hline & & Oral & Nasal & Oral & Nasal \\
\hline \multicolumn{2}{|r|}{ raíz } & $t i$ - 'cortar' & $\tilde{n}$ - 'comer' & ks- 'cantar' & $\underline{i} n$ - 'jugar' \\
\hline \multirow{4}{*}{ 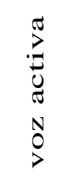 } & infinitivo & tiä/tiö & $\tilde{n} \underline{a}$ & 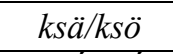 & $\underline{i} n \underline{a}$ \\
\hline & imperfectivo & $t i \ddot{e}$ & $\tilde{n} \underline{e}$ & $k s \dot{a} / k s \ddot{o}$ & $\underline{i} n \underline{a}$ \\
\hline & perfectivo & tiá & $\tilde{n} \underline{a}$ & $k s a ́$ & $\underline{i} n \underline{a}$ \\
\hline & imperativo & tió & $\tilde{n} \underline{o}$ & $k s \ddot{o}$ & $\underline{\text { innó }}$ \\
\hline
\end{tabular}


En cabécar, los verbos intransitivos, de forma prototípica, solo requieren la presencia de un único argumento, el cual toma por defecto el caso absolutivo. Esta categoría es la función sintáctica más básica en cabécar y por ello no lleva ningún tipo de marca, a excepción de que, de forma forzosa, debe anteceder al verbo. Al respecto, considérese la oración (1).

\section{(1) Wíshka kap-á=wa . \\ gato dormir-pfv-aft \\ 'El gato se durmió.'}

Sin embargo, algunos verbos intransitivos de movimiento ( $d \ddot{a}$ / dö 'llegar', bitälbitö 'venir' y mi $\underline{i} a$ ' 'ir') agregan un participante al evento, con el fin de señalar que el objeto del que se habla no llega de forma fortuita, sino gracias a la intervención de un actor externo, que se marca con la posposición $w \underline{a}^{19}$. Tal es el caso de (2).
Q Tsirú bit-é s'wa.
cacao venir-pfv $1 \mathrm{sg}$ met
'Traje cacao.'

Los verbos transitivos presentan una situación extralingüística en la que alguna entidad realiza una acción que afecta a otra entidad. El participante que tiene el control de la acción se marca con el caso

19 Además, como se observa en (2), los verbos de movimiento, a pesar de ser intransitivos, tienen la facultad de flexionarse con el sufijo -ë^-é, el cual, en este caso, tiene un sentido de perfectividad. Con respecto a la posposición wa , en un trabajo anterior, se concluyó que este elemento designa a un elemento oracional que fundamentalmente participa de la situación expresada por el verbo sin tener completo control de ella y por ello es inapropiado aplicarle la etiqueta de ergativo. Es

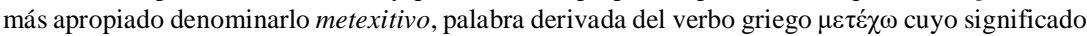
se relaciona con las ideas de 'participar, tener parte o participación (en algo), formar parte de, estar entre, recibir como parte o participación'. Al respecto, véase Guillermo González Campos, «Marcado diferencial de agente en cabécar», ponencia presentada en el I Congreso Internacional de la Asociación Centroamericana de Lingüística, Guatemala, 2011. 
ergativo; la otra entidad va en caso absolutivo, como puede apreciarse en (3), (4) y (5).
(3) $S a$
te tsirú $y$-á
tái.
1 pl.incl erG cacao beber-pfv mucho
'Bebimos mucho chocolate.'

( ) Bulía yís te $i$ sh-ё ijé $\underline{i} a$

. mañana $1 \mathrm{SG}$ erG 3 decir-ipfv 3SG dat

'Mañana le digo.'

(5) Sịna te kal julá buts-á.

viento erG árbol brazo romper-pfv

'El viento partió la rama del árbol.'

Obsérvese que la posposición te/të que marca el ergativo tiene la característica de aparecer únicamente con las formas verbales imperfectivas y perfectivas ${ }^{20}$. En el imperativo, existe una marca especial de ergativo. Se trata de la posposición $k \underline{u}$, cuyo uso se puede apreciar en (6).

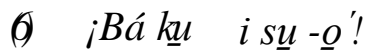 \\ 2sG erG 3 ver-imp \\ ¡Véalo!}

En cabécar, los verbos suelen ser intransitivos o transitivos. No obstante, algunos verbos presentan la facultad de conjugarse de ambas formas. Uno de ellos es pákä/pákö 'contar, narrar', el cual, como se aprecia en (7) y (8), posee un uso transitivo y otro intransitivo.

20 Las formas verbales perfectivas son, en cabécar, intrínsecamente afirmativas. La negación de estas formas se construye con el perfectivo negativo de voz media. 
Ø) Jé kë́képa ka pák-ä.

dem anciano mundo narrar-ipfv

Ese mayor cuenta historias.

(8) Yís te tulu pák-ë.

1SG erG luna narrar-ipfv

Yo cuento la historia de la luna.

El retrospectivo de la voz activa y la flexión de voz media se construye $-n$ en cabécar sobre la base de la raíz verbal fuerte. El primero se forma agregándole a esta raíz el sufijo - $l \sim l e ́$. La voz media, por su parte, se constituye adicionando el sufijo $-n \sim-r$ (sonidos son alófonos de /d/ en cabécar) y luego, en algunos casos, una desinencia adicional, que siempre es nasal. En resumen, los sufijos flexivos que se agregan a la raíz fuerte se presentan en el cuadro 9 .

\section{Cuadro 9. Desinencias verbales del cabécar que se agregan a la raíz fuerte}

\begin{tabular}{|c|c|c|}
\hline $\mathbf{v a}$ & retroSpectivo & $-l \sim-l \ddot{e}$ \\
\hline \multirow{6}{*}{ 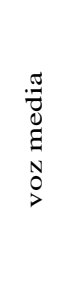 } & infinitivo & $-n+-\underline{a}$ \\
\hline & imperfectivo & $-r$ \\
\hline & perfectivo & $-n+-\underline{a}$ \\
\hline & perfectivo neGativo & $-n+-\underline{e}$ \\
\hline & imperativo & $-n+-\underline{o}$ \\
\hline & retroSpectivo & $-n+-\underline{e}+-l \sim-l \ddot{e}$ \\
\hline
\end{tabular}

Del cuadro 9, señalamos dos aspectos. El primero es que de acuerdo con nuestras observaciones, la forma de imperativo medio solo se presenta en Chirripó. En las demás áreas dialectales, el imperativo medio es cubierto por el perfectivo medio. El segundo es que existe una cantidad significativa de verbos que solo poseen raíz fuerte y, por ende, se conjugan únicamente según el esquema anterior. 
Entre ellos, se encuentra sé na 'vivir', cuya conjugación se presenta en el cuadro 10 a modo de ejemplo.

\section{Cuadro 10. Ejemplo de conjugación de las formas verbales cabécares construidas a partir de la raíz fuerte}

\begin{tabular}{|c|c|c|}
\hline \multicolumn{3}{|c|}{$s \underline{e}$ - 'vivir' } \\
\hline va & retroSpectivo & sélë \\
\hline \multirow{6}{*}{ 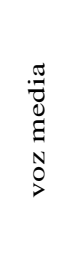 } & infinitivo & sé $n \underline{a}$ \\
\hline & imperfectivo & $s \underline{e} r$ \\
\hline & perfectivo & sé $n \underline{a}$ \\
\hline & perfectivo neGativo & sé $n \underline{e}$ \\
\hline & imperativo & sénó \\
\hline & retroSpectivo & $s \underline{e} n \underline{e} l$ \\
\hline
\end{tabular}

Los clíticos verbales

El último elemento que conforma el verbo cabécar son los clíticos verbales ${ }^{21}$. Como en otras lenguas, estas formas gramaticales se caracterizan por carecer de independencia fónica. En cabécar, se agregan a los verbos para crear matices de tipo aspectual y modal. Por ejemplo, como se vio antes, el clítico - $m \underline{i}$ al agregársele a una forma verbal en perfectivo da el sentido de aspecto incoativo. Al respecto, compárese la expresión $i$ ksá 'cantó' con $i$ ksámi 'empezó a cantar'. No obstante, si le agregamos este mismo clítico a una forma verbal en imperfectivo, se añade el significado de potencialidad de la acción. De esta forma, $i$ ksä́/i ksö́ 'canta' se vuelve $i$ ksä́mi / $i$ ksö́mi 'puede cantar'.

En el caso anterior, $-m \underline{i}$ varía su significado dependiendo de la forma verbal a la que se agregue. Un caso similar es el de -wa .

21 Los clíticos verbales son tema de controversia dentro de la lingüística. No es este el espacio para discutir, desde un punto de vista teórico, sobre ellos. Al respecto, véanse las consideraciones de $\mathrm{R}$. M. W. Dixon y Alexandra Y. Aikhenvald (Eds.), Word: A Cross-Linguistic Typology (Cambridge University Press, 2002) y de Stephen R. Anderson, Aspects of the Theory of Clitics (Oxford: Oxford University Press, 2005). Tampoco se puede hacer aquí una descripción exhaustiva de su funcionamiento en cabécar, tarea que será llevada a cabo en un próximo artículo. 
Cuando se agrega a una forma imperfectiva denota modalidad desiderativa, tal y como puede apreciarse en oraciones como (9) y (10).

(9) Yis te café $y-\ddot{e}=w a$.

$1 \mathrm{SG}$ erG café beber-ipfv $=$ deS

'Tengo ganas de tomar café.'

(1) Wilson te Alice $\underline{s u}-\underline{e}=w \underline{w a}$.

Wilson erG Alice ver-ipfv= $\mathrm{deS}$

'Wilsonquiere vera Alice.'

Sin embargo, si este mismo clítico se adiciona a una forma verbal perfectiva, el significado que transmite es el de afectación total del elemento nominal que ocupa la posición de absolutivo. Esto puede apreciarse en (11) y (12).

(II) Yis te café $y-\dot{a}=w \underline{w}$.

$1 \mathrm{sG}$ erG café beber-pfv=aft

'Me tomé todo el café.'

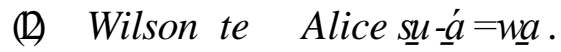

Wilson erG Alice ver-pfv=aft

'Wilson vio a Alice.' (Vio todo lo que ella hizo).

Otros clíticos nunca cambian su significado; por ejemplo, $-n \underline{i}$ siempre significa 'reiteración', sin importar si se agrega a formas perfectivas o imperfectivas. Esto puede verse en (13) y (14).

(B) Yís tú $-n \underline{-a}=n \underline{i} \quad y i k i ́$.

$1 \mathrm{sg}$ correr-med-rep ayer

'Ayer corrí otra vez'. 
(A) Yís tiú-r=ni bulía.

1sG correr-med-rep mañana

'Mañana corro de nuevo'.

En este caso, se trata de un elemento cuya incidencia sobre la significación del verbo no es tan directa, prueba de ello es que, de existir varios clíticos verbales, siempre ocupa la posición final. Un futuro estudio deberá examinar con atención este tipo de fenómenos y confirmar la existencia de órdenes de inserción y sus consecuentes niveles de incidencia en la estructura de estos clíticos verbales.

En cabécar, los clíticos son elementos morfológicos que, por lo general, son opcionales. No obstante, hay verbos que, por su significado, requieren la presencia casi obligatoria de un clítico en específico. Tal es el caso de bikäsa 'cortar en pedazos' y däju 'llegar'. Aun en casos como estos, es factible encontrar construcciones sintácticas en las que no aparece el clítico, como puede verse en (15).
(5) Ijé $d$-ä rä bulílí bulílí.
3sg llegar-ipfv ads diario diario
'Él llega todos los días'.

Algunos verbos cambian su significado dependiendo del clítico con que se construyan; por ejemplo, en Chirripó, el verbo ktä unido al clítico - wa significa matar, como en (16).

(16) Urí te Rogelio kt-á=wa .

oso.caballo erG Rogelio dañar-pfv-aft

'El oso caballo mató a Rogelio'

Este mismo verbo unido al clítico $-s \underline{a}$ denota el proceso de 'enfermarse' (17). 
(I) Jálibá te Rogelio kt-á=sa . reumatismo erG Rogelio dañar-pfv-afp

'Rogelio se enfermó de reumatismo'.

Existen algunos clíticos que marcan la pluralidad del elemento nominal que ocupa la posición de absolutivo, como puede apreciarse en (18), (19), (20) y (21).

(8) Aláklä $d-\ddot{e}=\underline{j u}$.

mujer llegar $-\mathrm{pfv}=\mathrm{dir}=\mathrm{pl}$

'La mujer llegó.'

(9) Aláklä-wá $d-\ddot{e}=j \underline{j} u=l \underline{u}$.

mujer-pl llegar- $\mathrm{pfv}=\mathrm{dir}=\mathrm{pl}$

'Las mujeres llegaron.'

(a) Wíshka te ko ño kt-á=wa .

gato erG ratón dañar-pfv $=$ aft

'El gato mató al ratón.'

(2) Wishka te ko $k t-a ́=c h \underline{a}=w \underline{a}$.

ño

gato erG ratón dañar-pfv=pl=aft

'El gato mató los ratones.'

Finalmente, obsérvese que este tipo de elementos morfológicos tiene la capacidad de combinarse para crear significados complejos. Compárense, al respecto, (22) y (23).

(2) ¿Bá te ulúk ti-á?

2SG erG cedro cortar-pfv

¿¿Usted cortó el cedro?' 


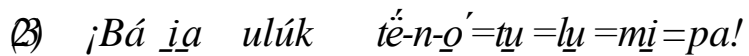

$2 \mathrm{sG}$ dat cedro cortar-med-imp $=\mathrm{dir}=\mathrm{pl}=\mathrm{inc}=\mathrm{yuS}$

‘¡Ojalá cortara (usted) los cedros!'

Al combinarse, los clíticos pueden intercambiar de posición generando cambios de significado, como se puede apreciar en las oraciones (24), (25), (26) y (27).

(24) I $d u-\ddot{a}=w \underline{a}=m \underline{i}$.

3 morir $-\mathrm{ipfv}=\mathrm{aft}=$ pot

'Está a punto de morir.'

(2) I $d u-\ddot{a}=m \underline{i}=w \underline{a}$.

3 morir-ipfv $=$ pot $=$ aft

'Se va a morir.' (Es seguro, pero no se sabe cuándo).

(4) Ka $\underline{\underline{l}} \underline{i} \quad j \underline{a}-r=w \underline{a}=m \underline{i}$.

Lluvia caer - med $=\mathrm{aft}=$ pot

'Yacasi empieza a llover.'

(2) $K \underline{a} \underline{l i} \quad j \underline{j a}-r=m \underline{i}=w \underline{a}$.

Lluvia caer-med $-=$ pot $=$ aft

'Va a llover.' (Se ven las nubes oscuras).

Estos elementos constituyen, por tanto, un aspecto de la gramática del cabécar que debe estudiarse más pormenorizadamente. Debe hacerse un inventario completo de los clíticos verbales del cabécar, y estudiar a profundidad sus propiedades distribucionales y los significados que transmiten a la significación primaria denotada por el verbo conjugado. 


\section{Abreviaturas}

$\begin{array}{llll}1 & \text { primera persona } & \text { inc } & \text { incoativo } \\ 2 & \text { segunda persona } & \text { ipfv } & \text { imperfectivo } \\ 3 & \text { tercera persona } & \text { med } & \text { voz media } \\ \text { adS } & \text { adscriptivo } & \text { met } & \text { metexitivo } \\ \text { aft } & \text { afectación total } & \text { pfv } & \text { perfectivo } \\ \text { dat } & \text { dativo } & \text { pl } & \text { plural } \\ \text { dem } & \text { demostrativo } & \text { pot } & \text { potencial } \\ \text { deS } & \text { desiderativo } & \text { rep } & \text { repetitivo } \\ \text { dir } & \text { direccional de movimiento } & \text { SG } & \text { singular } \\ \text { erG } & \text { ergativo } & \text { yuS } & \text { yusivo } \\ \text { imp } & \text { imperativo } & & \end{array}$

